\title{
Die Verantwortung des Verbrauchers und einige ihrer Grenzen
}

\author{
Peter Kunzmann
}

Eingegangen: 31 August 2009/Angenommen: 10 September 2009/Online publiziert: 8 January 2010 (c) Birkhäuser Verlag, Basel/Switzerland 2009

Ein typischer Fall: Die Sendung „Monitor“ zeigt einen Bericht über angebliche Missstände in bayerischen Putenmastbetrieben. Sonja Mikich moderiert lakonisch: „Der Verbraucher will es eben billig“. Was für ein bemerkenswerter Kommentar! Wie selbstverständlich wird der Verbraucher zum eigentlich Schuldigen für Zustände, die er nie gesehen, nie gebilligt oder gar gewollt hat. Schuldig wird er in seiner gedankenlosen Geiz-ist-geil-Mentalität, deren katastrophale Folgen unschuldige Truthühner tragen müssen. Der gehobene Zeigefinger deutet nicht auf die unmittelbar Handelnden, in diesem Falle also die Tierhalter, sondern auf den eigentlich Verantwortlichen: Der Verbraucher kauft Fleisch aus der „Massentierhaltung“, betreibt Kühlschränke ohne Rücksicht auf ihren Energieverbrauch, fliegt gegen jede ökologische Vernunft in den Urlaub statt im Sauerland zu radeln, und tränke seine Cola heute immer noch aus der Dose, wäre ihm das Pfand nicht zu teuer geworden. Verantwortlich ist derjenige, auf den alle Missstände letztlich zurückzuführen sind, weil er am Ende jeder Kette von Waren und Dienstleistungen mit Geld honoriert, was immer geschieht: eben der Verbraucher.

Tierschutz in der Landwirtschaft ist ein ideales Beobachtungsfeld für eine eigenartige Zuschreibung von Verantwortung, die letztlich auf die Aufhebung von Verantwortung hinausläuft. Wo immer Missstände in der Nutztier-Haltung inkriminiert werden, ist der Verbraucher der letzte Dominostein, der fällt.

Prof. Dr. P. Kunzmann ( $\square)$

Universität Jena, Lehrstuhl für Angewandte

Ethik/Ethikzentrum, Zwätzengasse 3, 07743 Jena, Germany

e-mail: Peter.kunzmann@uni-jena.de
Die Reihe beginnt bei der konkreten Anklage gegen bestimmte Zustände. Der Tierhalter zuckt mit den Achseln. Er würde ja seinen Tieren gerne Besseres gewähren, wenn er es bezahlt bekäme. In den Worten eines Landwirtschaft-Funktionärs: „Wenn es bezahlt würde, bänden wir den Schweinen rosa Schleifchen um." Aber der Landwirt sieht sich unter ökonomischen Druck durch den Handel, der seinerseits auf den Einzelhandel verweist und dieser wiederum auf den Verbraucher: Wäre der Verbraucher nur ein wenig einsichtiger und verantwortungsbewusster, gäbe er nur ein wenig mehr aus - alles wäre gut. Genau hier ist die Verantwortung schon verpufft, denn diejenigen, die unmittelbar ins Geschehen eingreifen, sind schon als diejenigen entschuldigt, die eigentlich Opfer der Umstände geworden sind. Die Verantwortung scheint bei dem zu liegen, der eigentlich am weitesten von der moralisch belasteten Situation entfernt agiert. Nur diesen letzten Dominostein müssen wir wieder aufrichten, und alles kommt ins Lot!

Warum geht das nicht? Erste klare, triviale Antwort: weil es den Verbraucher nicht gibt! Der Verbraucher sind die Verbraucher, circa 80 Millionen von ihnen allein in Deutschland, sehr verschieden in ihren Verhältnissen und ihrem Verhalten, sehr verschieden in Einkommen und Ausgaben. Die Rede von „der Verbraucher“ suggeriert, wir hätten es mit einem einzigen Akteur zu tun. Doch schon das Beispiel mit der Nutztier-Haltung zeigt, dass der Verbraucher es nicht notwendig „nur billig haben will“, gibt es doch unter den Verbrauchern auch den Biomarkt-Gourmet und auch den Vegetarier.

Bei der Frage nach Macht oder Ohnmacht des Verbrauchers dürfen wir festhalten: die Ohnmacht des Verbrauchers erweist sich darin, dass die gängige 
Redensart ein Handlungs-Subjekt suggeriert, das so überhaupt nicht existiert. Der Verbraucher existiert nicht, weil er nicht als einzelner handelt. Die Verbraucher handeln, aber eben nicht in dem Sinne, wie z. B. die Tierhalter handeln oder eben auch ein Konzern. Die Verbraucher handeln natürlich im Plural; doch das tun sie nicht in der gleichen Weise, wie andere „Player“ nicht als Individuen agieren, sondern zu mehreren. Philosophisch kann man dies etwas genauer zeichnen, wenn man sich verschiedene Typen von Handlungskausalität vor Augen hält. Die wahren Experten für die Analyse des Zusammenwirkens verschiedener Akteure sind - nebenbei bemerkt - wohl die Strafrechtler, denn sie müssen bei gemeinschaftlich begangenen Taten einen Weg finden, den beteiligten Individuen die individuelle schuld zuzuschreiben.

Für die moralische Bewertung ist sehr grob korporatives, kooperatives und kollektives Handeln zu unterscheiden: (1) Annäherungsweise lässt sich korporatives Handeln dadurch kennzeichnen, dass jeder Beteiligte in einem arbeitsteiligen Vorgang eine ihm genau zugewiesene Funktion erfüllt. In diesem Sinne sind beispielsweise Firmen und Unternehmen korporative Akteure, die bestimmte Effekte dadurch zu Stande bringen, dass jeder, vom Vorstandsvorsitzenden bis zum Pförtner, an einer bestimmten Position zum Handlungserfolg des Apparats beiträgt - dem Erfolg, der gefährdet ist, wenn auch nur eine Funktion ausfällt. Ein instruktives, wenn auch schauerliches Beispiel bietet das juristische Nachspiel bei Katastrophen wie dem Zugunglück von Eschede oder der eingestürzten Eissporthalle in Bad Reichenhall: jeder Akteur war auf seine Weise beteiligt, und hätte nur einer von ihnen anders gehandelt, wäre das grauenvolle Ergebnis nicht Realität geworden - ein Ergebnis, dass schließlich keiner gewollt hat. (2) Kooperatives Handeln dagegen ist dadurch ausgezeichnet, dass der Effekt dadurch zu Stande kommt, dass viele Beteiligte Handlungen desselben Typs vollziehen, wobei sie untereinander austauschbar sind, aber auf ein Ziel gerichtet sind. Mehrere freundliche Helfer schieben ein liegen gebliebenes Auto an; mehrere Hooligans vom Club A verprügeln einen Hooligan vom Club B. Hübsche Beispiele für kooperatives Handeln geben z. B. alle Arten von Kartellen ab. (3) Das kollektive Handeln wiederum ist davon dadurch unterschieden, dass der Effekt zu Stande kommt, ohne dass er im Handeln des einzelnen angelegt oder intendiert ist. Für kollektive Effekte wäre das Paradigma der Stau: Er entsteht ohne das Wollen der Einzelnen, in diesem Fall sogar gegen ihre Interessen; der Beitrag des Einzelnen ist nicht ausschlaggebend und selbst wenn der Einzelne sich entzieht, ändert dies nichts am Ausgang des Ganzen.

Die Macht des Verbrauchers beruht nun ganz wesentlich auf Effekten, die er nur durch kollektives Handeln bewirkt. Das klingt banal, hat aber für die Bestimmung auch der Verantwortung des Verbrauchers einige Konsequenz.

Ich definiere Verantwortung als Zuschreibung von Handlungsfolgen unter Wertgesichtspunkten.

Diese Definition drückt aus, dass die Verantwortung für etwas nicht einfach besteht, sondern in je eigenen Akten eigens bezeichnet, zugeschrieben wird. Verantwortung hält fest, dass irgendwer - sei es ein Individuum, eine Gruppe, ein Kollektiv, eine Organisation - an der Entstehung von Sachverhalten mitgewirkt hat, die einer Bewertung unterliegen. Ohne einen Werthorizont halten wir bestenfalls Kausalität fest, aber nicht Verantwortung. Wir unterstellen, dass das, wofür wir jemanden verantwortlich machen oder zur Verantwortung ziehen, in mindestens einer Perspektive relevant und nicht gleichgültig ist, z. B. rechtlich, moralisch, wirtschaftlich, sozial. Für die Dimension von Verantwortung wird als Handlung umgekehrt nur interessant, was bewertbare Folgen hinterlässt oder hinterlassen kann; alles andere zeichnet nur Kausalität nach. Der Handlungsstrang selbst verknüpft den Verantwortlichen mit dem Gegenstand und dem Grund seiner Verantwortung, im Idealfall eineindeutig.

Diese Relation aber finden wir selten, wenn der Verbraucher in einer Reihe mit anderen Akteuren wie eben den Produzenten oder auch den Überwachungsorganen verantwortlich gemacht werden soll, auch wenn sich entsprechende Appelle zum Beispiel in Kirchenkreisen ganz anders anhören: Dem einzelnen Verbraucher sind Handlungsfolgen nicht im korporativen Sinne zuzurechnen, da seine individuellen Entscheidungen im Großen folgenlos bleiben. Das mag trivial klingen, aber vor diesem Hintergrund verbieten sich Sprachspiele, in denen der Verbraucher in einer Linie mit anderen oder gar letzten Endes politisch oder moralisch verantwortlich gemacht wird, dort, wo seine individuelle Handlungsmacht nicht hinreicht. Es ist richtig: Manche Missstände wären nicht möglich, wenn ihre Produkte keine Abnehmer fänden, auch unter den deutschen Verbrauchern. Den Verbrauchern. Weil aber die Zustände nicht vom handelnden, vom kaufenden und konsumierenden Individuum abhängen, hat es auch keinen Sinn, den moralischen Zeigefinger auf dieses Individuum zu richten. 
Das ändert sich freilich, wenn es interessierten Verbrauchern gelingt, ihre nur kollektive Handlungsmächtigkeit in eine kooperative oder gar korporative $\mathrm{zu}$ wandeln, etwa durch die enorm gestiegenen Möglichkeiten, durch alle möglichen Formen von öffentlichen Bewertungen, Erfahrungen über Kaufentscheidungen und Kauf-Fehlentscheidungen zu bündeln oder auch entsprechende Institutionen zu etablieren, die aus dem unkoordinierten Nebeneinander von einzelnen Kausalitäten etwas wie den Verbraucher formen können.

Das Gesagte darf nicht missdeutet werden als eine Generalsabsolution: Natürlich behält der Verbraucher seine Verantwortung, aber eben nur in der Reichweite, die er selber mit seinem Handeln formt. Wenn er auch mit seiner einzelnen Aktion oder auch seinem individuellen Verzicht nicht für das Ganze haften kann, bleibt es doch bei seiner einzelnen zurechenbaren Handlung, im letzten bei seinem unvertretbaren Gewissensentscheid. Um am Beispiel der Tierhaltung zu bleiben: Wer etwa zur Überzeugung gelangte, bestimmte Produktionsformen tierischer Erzeugnisse nicht unterstützen zu wollen, ist moralisch gehalten, für sich selbst die Konsequenzen zu ziehen, auch wenn er damit das große Ganze dieser Produktionsformen nicht beeinflusst. Der Hinweis, das eigene Verhalten ändere doch nichts, leistet keine moralische Rechtfertigung; Konsequenz als Ausrichtung des Handelns an den eigenen Überzeugungen ist immer noch eine sittliche Tugend. Ihr alter Name war Tapferkeit.

Doch dies setzt Einsicht in das Richtige voraus. Der Idealfall ist der Konsument, der eine informierte Wahl trifft. Die Frage muss erlaubt sein, wie weit der Verbraucher verantworten soll, was er so gar nicht verstehen kann. Schon hier zeigen sich mehr Probleme, als die landläufigen Appelle an die so genannte „Eigenverantwortung“ üblicherweise aussprechen, setzen sie doch einen Grad von Informiertheit voraus, der aus einer ganzen Reihe von Gründen illusionär erscheint.

Wie tief eigentlich darf man diesen Grad von Informiertheit ansetzen? In der Medizinethik gibt es den Ausdruck des „informed consent“ (oder moderner: „informed choice“), der Zustimmung nach Aufklärung. Wenn der Arzt den Patienten in einer Weise informiert, dass dem Patienten vernünftigerweise nichts anderes übrig bleibt, als in die vorgeschlagene Therapie einzuwilligen, nennt man dies spöttisch „informed consent under duress“. In diese Situation kommen wir alle als Verbraucher, wenn uns der Kfz-Meister erklärt, unser Wagen brauche eine neue Lenkscheibe, oder der Monteur, unsere Waschmaschine sei nun irreparabel defekt.

Scheinbar aber haben wir in vielen anderen Fällen einen höheren Grad von Wahlfreiheit; aber auch dieser consent hängt an der Information und genau hier endet die eigentliche Verantwortlichkeit des Verbrauchers, weil ihm nur in sehr engen Grenzen zuzumuten ist, sich diese Information zu verschaffen.

Wir haben es in der modernen Lebenswelt mit außerordentlich komplexen Phänomenen zu tun, und zwar allerorten, die der einzelne nicht überblicken, verstehen und angemessen bewerten kann. Er ist damit in den vielen Entscheidungen, die er treffen muss, von Experten abhängig; und dies, so meine These, tiefer, als es den Anschein hat. Denn selbst da, wo Systeme darauf ausgerichtet sind, dem Verbraucher mehr eigene Entscheidungsräume zu schaffen, steht dahinter Expertenwissen. Die Funktion solcher Expertenkreise lässt sich ebenso umständlich wie zutreffend als Komplexitätsreduktion bezeichnen. Der Experte übersetzt für den Laien aus jener Welt, in die hinein dieser handeln soll, ohne recht zu wissen, was er da tut. Alle Entscheidungen, die solchermaßen in komplexe Gefüge hinein getroffen werden, sind in gewisser Hinsicht „unverantwortlich“. Streng genommen erhöht sich nicht die wirkliche Freiheit des Verbrauchers, sondern der Verantwortungs-Rahmen des Experten, dem er nun vertrauen muss. Ein Muster dafür sind Labelingsysteme. Die Experten nehmen dem Verbraucher die Kriterien aus der Hand, nach denen er bewerten müsste, was er nicht beurteilen kann. Ein Ampelsystem für die ernährungswissenschaftliche Qualifizierung von Fertigprodukten erklärt dem Verbraucher nicht mehr, was in den Lebensmitteln steckt, sondern was er davon zu halten hat.

Die Alternative bestünde darin, jede Menge Daten auf die Verpackung zu drucken und es dem Käufer $\mathrm{zu}$ überlassen, welchen Reim er sich darauf macht. Doch auch diese Alternative ist keine echte, denn wie viel an Uran darf Grundwasser enthalten und wie viele Milligramm Magnesium brauche ich täglich?

Die Deutungshoheit des Experten ist nicht $\mathrm{zu}$ überschlagen und dem Verbraucher bleibt nichts als ihm zu vertrauen. Wenn Thilo Bode beispielsweise öffentlich erklärt, wie gefährlich das Uran im Trinkwasser „wirklich“ sei, wird es kaum jemanden geben, der mit ihm über Mikrogramm oder ppm rechten wird. Und doch legt man dem Publikum die Grenzwerte und ihre Überschreitung quantifiziert vor, als ob die Verbraucher im Ernst eine Chance hätten, sich darüber selbstständig ein Urteil zu bilden. 
Das ist fair, solange es nicht mit dem Gestus einhergeht, ein jeder sei in diesen Angelegenheiten doch informiert und ergo seines Glückes Schmied. In Wirklichkeit liegt die Verantwortung bei jenen Experten, unter deren Deutung aus den Zahlen und Fakten die Begriffe und Sachverhalte werden, die für die Verbraucher handlungsleitend sind. Die Verantwortung, die Experten damit auf sich nehmen, ist damit von großer moralischer Relevanz und verlangt neben bestmöglicher Sachkenntnis auch ernste persönliche Integrität.

Für die Verbraucher verschieben sich damit die Abhängigkeiten und - wenn meine These richtig ist erklärten sich eine ganze Reihe von Phänomenen zumindest teilweise daraus, dass hier eine Kluft entsteht zwischen dem, was Individuen als Verbraucher eigentlich verantworten sollen, und dem, was sie auf Wissen basiert entscheiden können.

Eine Strategie bzw. ein hilfreicher Ausweg ist, sich auf das vermeintlich Klare zu stürzen. Das erklärt den außerordentlichen Rang, in den einige besondere Symbole im öffentlichen und im politischen Diskurs gerückt wurden. Was, so könnte man fragen, motiviert eigentlich eine ansonsten in Tierschutzfragen nicht so arg engagierte deutsche Öffentlichkeit, mit Verve gegen die Käfighaltung von Legehennen zu opponieren? Teil einer möglichen Antwort: Hier sieht sich der Verbraucher in einem der ganz seltenen Fälle, wo die Alternativen strikt zweiwertig - ja oder nein - und für ihn einsehbar vor ihm stehen. Dass Hennen in Käfigen gehalten werden, ist selbst für den Laien gut zu erkennen, im Unterschied zu den tausend anderen Fragen, mit denen sich Nutztierhalter befassen müssen: Besatzdichten, Lüftungssysteme, Transportzeiten, Medikation, Spaltenbreiten etc. etc. Wer also überhaupt Verantwortung für die Herkunft seiner tierischen Nahrungsmittel in sich verspürt, hat einen der wenigen Streitfragen mit scharfen Kanten vor sich. Das gilt mutatis mutandis auch für die Grüne Gentechnik: Auch im Ackerbau hat der Laie selten die Chance, zwischen Ja und Nein, zwischen Schwarz und Weiß zu urteilen - Gentechnik, ja oder nein? - das scheint doch einmal eine übersichtliche Angelegenheit.

Eine echte Strategie, der Komplexität aus dem Weg zu gehen, ist die Flucht ins Idyll und ins Einfache. Wo der Expertenstreit über beinahe alles in der Welt verwirrt und verunsichert, etabliert sich die Gegenwelt des Bukolischen, die mit ihren Großmutterrezepten und ihrem traditionellen oder pseudotraditionellen Gegenwissen um so üppiger sprießt, je unübersichtlicher das moderne Lehrbuch-Wissen wuchert. Ein Ausweg aus dem lähmenden Expertenstreit über die richtige Erziehung, die richtige Ernährung und die richtige Heilung ist der Rekurs auf einen Gegenentwurf, der gerade nicht das brandneue Forschungsergebnis kennt, sondern das uralte Wissen. Hierin liegt ein eigener Weg möglicher Vereinfachung sowohl der Inhalte wie des Mediums: Die Kelten (Hexen, Omas, Indianer...) haben nicht diskutiert und differenziert, sondern ganz einfach gewusst, wo es langgeht. Wenn meine Deutung richtig ist, lebt ein Teil dieser Gegenkultur aus der Über-Spannung zwischen mannigfachen Entscheidungszwängen und der Unbeherrschbarkeit des eigentlich dazu nötigen Wissens.

Oder die Unschlüssigen binden sich an denjenigen, der für sie und an ihrer Statt weiß. Wo es komplexer bleibt, wird das Ganze zu einem Spiel um Vertrauen und dieses Vertrauen genießen ausgewählte NGOs. Die Funktion von NGOs in heutigen Industriegesellschaften resultiert nicht zuletzt aus ihrer Leistung in der Komplexitätsreduktion; ihnen wird zugetraut, dass sie gleichsam tugendhaft genug sind, die Klugheit der Sachkunde mit der Lauterkeit zu verbinden, diese Klugheit nur zum Wohle der Allgemeinheit zu gebrauchen. Damit sind sie die Leuchttürme im sonst verwirrenden Durcheinander konkurrierender Darstellungen, die überdies immer interessengeleitet sind.

Kann „der Verbraucher“ eigentlich anders? Kann er sich eigentlich so verhalten, wie ihn die Medien gerne beraten - was bei ihnen "Service“ heißt nämlich sich gründlich informieren, vergleichen und kritisch die Augen aufhalten? Selbst wenn er dazu in der Lage wäre, sich in einen Bereich so zu vertiefen, dass er ohne Beistand aus sich beurteilen lernt, was für ihn und seine Entscheidungen relevant ist - für wie viele Bereiche eines durchschnittlichen Verbraucher-Lebens kommt ein solcher echter ,informed consent" überhaupt in seine Reichweite?

Der Verbraucher steht nämlich in der Schnittstelle ganz verschiedener Handlungskreise, bei ihm kollidieren immerzu ganz verschiedene Erfordernisse der Rollen und Funktionen. Die ganz „normale“ Dichte von Anfragen an seine Urteilsfähigkeit und Entscheidungskraft lässt es gar nicht zu, dass er außer einigen Stichproben gründliche Recherchen anstellt und außer einigen ihm besonders wichtigen Dinge ernstlich auswählen kann. Wieviel begründete Verantwortung kann der Konsument denn übernehmen, neben dem Vergleich von Handytarifen, Versicherungsmodellen, Bio- und Öko-Labels, Testergebnissen für seine nächsten Reifen unter Beachtung der Winterreifenverordnung? 
Die Mündigkeit, die eine Verantwortung des Verbrauchers erst begründen würde, hat ihre Grenze an der lebensweltlichen Übersättigung mit verbraucherrelevanten Vorgängen, die im Modus eines

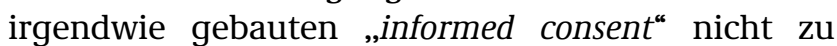
bewältigen sind. Das alles ist nicht dramatisch, sollte aber ein letzter und besonders wichtiger Grund sein, nicht „dem Verbraucher“ allzu forsch Verantwortung zuzuschreiben. Auch deshalb, weil sie woanders zu lokalisieren ist, wenn „der Verbraucher“ als ihr Träger nicht eigentlich in Frage kommt: bei
Produzenten, dem Handel, bei Behörden. Wenn am Ende der langen Reihe von Verantwortlichkeiten der Verbraucher genannt wird, dann heißt es nicht, dass von ihm aus auch alles andere gesteuert wird.

„Der Verbraucher will es eben billig?“ Vielleicht war der Verbraucher zu sehr mit anderen wichtigen Dingen befasst und hat keine besondere Ahnung von der Putenmast? Oder er ist deswegen nicht dafür verantwortlich, weil er ohnehin kein Geflügel isst, und selbst dies noch keinen Unterschied macht. 